\title{
Sex-specific effects of aging on humoral immune responses to repeated influenza vaccination in older adults
}

\author{
Janna R. Shapiro ${ }^{1}{ }^{1}$, Huifen $\mathrm{Li}^{2}$, Rosemary Morgan ${ }^{1}$, Yiyin Chen ${ }^{3}$, Helen Kuo ${ }^{1}$, Xiaoxuan Ning ${ }^{4}$, Patrick Shea ${ }^{5}$, Cunjin Wu ${ }^{6}$, \\ Katherine Merport ${ }^{7}$, Rayna Saldanha ${ }^{7}$, Suifeng Liu ${ }^{8}$, Engle Abrams ${ }^{2}$, Yan Chen ${ }^{9}$, Denise C. Kelly ${ }^{2}$, Eileen Sheridan-Malone ${ }^{2}$, Lan Wang ${ }^{10}$, \\ Scott L. Zeger ${ }^{11}$, Sabra L. Klein $\left(i D^{1,5 凶}\right.$ and Sean X. Leng $\mathbb{i D}^{2,5 凶}$
}

Older adults ( $\geq 65$ years of age) bear a significant burden of severe disease and mortality associated with influenza, despite relatively high annual vaccination coverage and substantial pre-existing immunity to influenza. To test the hypothesis that host factors, including age and sex, play a role in determining the effect of repeated vaccination and levels of pre-existing humoral immunity to influenza, we evaluated pre- and post-vaccination strain-specific hemagglutination inhibition (HAl) titers in adults over 75 years of age who received a high-dose influenza vaccine in at least four out of six influenza seasons. Pre-vaccination titers, rather than host factors and repeated vaccination were significantly associated with post-vaccination HAI titer outcomes, and displayed an age-bysex interaction. Pre-vaccination titers to H1N1 remained constant with age. Titers to H3N2 and influenza B viruses decreased substantially with age in males, whereas titers in females remained constant with age. Our findings highlight the importance of preexisting immunity in this highly vaccinated older adult population and suggest that older males are particularly vulnerable to reduced pre-existing humoral immunity to influenza.

npj Vaccines (2021)6:147; https://doi.org/10.1038/s41541-021-00412-6

\section{INTRODUCTION}

Seasonal influenza is an important public health burden in older adults (people $\geq 65$ years of age), particularly the oldest and frail subset $^{1-3}$. In the United States (U.S.), there are an estimated 4 million incident cases per year in older adults, accounting for $90 \%$ of deaths associated with influenza ${ }^{4,5}$. The U.S. Centers for Disease Control and Prevention (CDC) recommends annual influenza vaccination for prevention of influenza infection and complications in people 6 months and older 6 . The high-dose inactivated influenza vaccine (HD-IIV) is available to older adults and has demonstrated superior efficacy over the standard-dose vaccine in older age groups ${ }^{6,7}$. Seasonal influenza vaccination coverage is relatively high in older adults, with $>60 \%$ of older Americans being vaccinated annually, compared to $<40 \%$ vaccination coverage in the $18-49$ age group ${ }^{8}$.

Age-related immunosenescence, defined by a decline in cellular and humoral immune function combined with a chronic lowgrade inflammatory phenotype (CLIP), or inflammaging ${ }^{9-11}$, is believed to be the primary reason for the reduced effectiveness of influenza vaccines observed in older adults ${ }^{12-14}$. Repeated annual vaccination may also have a negative effect on vaccine-induced humoral immune responses as well as vaccine effectiveness (VE). For example, a recent observational test-negative study using ten years of vaccination history found that in older adults, VE decreases with increasing numbers of previous vaccinations but that vaccination continues to offer some level of protection ${ }^{15}$. Another study over eight seasons in the general adult population found that VE to H3N2, but not influenza B virus, is reduced among individuals with frequent vaccination history compared to those without prior vaccination ${ }^{16}$. Age-specific effects have also been observed in this context, with a reduction in influenza vaccine immunogenicity observed with repeat vaccination in teenagers, but not adults ${ }^{17}$. In addition, a meta-analysis found heterogeneous effects of repeated vaccination overall and that when negative effects are observed, they are most pronounced for $\mathrm{H} 3 \mathrm{~N} 2^{18}$. In contrast, a recent systematic review and metaanalysis concluded that the available evidence did not support a reduction in VE with consecutive repeat vaccination, but that certainty in the evidence was low ${ }^{19}$. Case-control studies in both Australia and Spain found beneficial effects of repeated annual vaccination on VE in older adults ${ }^{20,21}$, and an observational population-based study in Sweden found no differences in VE between those who had been vaccinated in the current season only and those who had been vaccinated in both the current and previous seasons ${ }^{22}$. Based on the conflicting evidence, multiseason clinical studies to address the effects of aging and repeated vaccination have been recommended ${ }^{18}$.

Mechanistically, pre-existing immunity generated to various influenza virus exposures over time can have an important impact on the outcome of vaccination. According to the immune imprinting theory, the memory response established by an individual's first influenza exposure has a lifelong effect on subsequent immune responses to infection or vaccination ${ }^{23}$. Broad pre-existing immunity is thought to have negative consequences, as pre-existing antibodies can suppress the

\footnotetext{
${ }^{1}$ Department of International Health, Johns Hopkins Bloomberg School of Public Health, Baltimore, MD, USA. ${ }^{2}$ Division of Geriatric Medicine and Gerontology, Department of Medicine, Johns Hopkins University School of Medicine, Baltimore, MD, USA. ${ }^{3}$ Guangdong Geriatrics Institute, Guangdong Provincial People's Hospital, Guangdong Academy of Medical Sciences, Guangzhou, Guangdong, China. ${ }^{4}$ Department of Geriatrics, Xijing Hospital, The Fourth Military Medical University, Xi'an, Shaanxi, China. ${ }^{5}$ W. Harry Feinstone Department of Molecular Microbiology and Immunology, Johns Hopkins Bloomberg School of Public Health, Baltimore, MD, USA. ${ }^{6}$ Department of Geriatrics, The Second Hospital of Tianjin Medical University, Tianjin, Hebei, China. ${ }^{7}$ Zanvyl Krieger School of Arts and Science, Johns Hopkins University, Baltimore, MD, USA. ${ }^{8}$ Zhongshan Hospital, Xiamen University, Xiamen, Fujian, China. ${ }^{9}$ Department of Geriatrics, The First Affiliated Hospital of Chongqing Medical University, Chongqing, Sichuan, China. ${ }^{10}$ Department of Geriatrics, The First Affiliated Hospital, Zhejiang University School of Medicine, Hangzhou, Zhejiang, China. ${ }^{11}$ Department of Biostatistics, Johns Hopkins Bloomberg School of Public Health, Baltimore, MD, USA. ${ }^{凶}$ email: sklein2@jhu.edu; sleng1@jhmi.edu
} 
response to novel influenza virus strains by reducing the amount of available antigen or epitope masking ${ }^{24,25}$. A theoretical benefit of HD-IIV is that pre-existing antibodies cannot sequester the increased amount of antigen delivered, and, thus, more antigen is available to activate memory $B$ cells and elicit a protective response ${ }^{26-28}$. To our knowledge, however, the impact of preexisting immunity in the context of HD-IIV has not been adequately characterized.

In addition to age, other host factors including sex, frailty, and body mass index (BMI) can impact vaccine responses in older adults. Females have been found to mount greater antibody responses to HD-IIV than males ${ }^{29}$. The immunological differences between males and females in immune responses are largely attributed to sex hormones and sex chromosomes ${ }^{30-32}$. Inflammaging also contributes significantly to age- and sex-related differences in immune responses and vulnerability to infections, as discussed in two recent reviews in the context of SARS-CoV-2 infection and ongoing pandemic ${ }^{31,33}$. The relationship between frailty and influenza vaccine responses is debated in the literature, with one study reporting frailty having a negative effect ${ }^{34}$, others reporting no effect ${ }^{35-39}$, and others still reporting a positive effect ${ }^{40}$. Finally, in older adults, obesity, as measured by $\mathrm{BMI}$, is significantly associated with decreased hemagglutination inhibition (HAI) titers and percentage of switched memory B cells ${ }^{41}$. Whether host related factors, including sex and age, explain variation in pre-existing immunity following repeated vaccination has not been reported. We hypothesize that the variation across studies in estimates of the effects of pre-existing immunity and repeated annual vaccination is partly caused by failure to adequately account for heterogeneity and interactions among host factors that likely differ across studies. To address this knowledge gap, we used a longitudinal cohort of older adults over 75 years of age who had received high-dose, trivalent inactivated influenza vaccine (HD-IIV3) in at least four out of six influenza seasons to estimate the impact of repeated vaccination on the antibody response to HD-IIV3 and its dependence on the intersection of age, sex, frailty, BMI, and pre-existing immunity.

\section{RESULTS}

\section{Study participants and annual influenza immunization with} HD-IIV3

Over the six influenza seasons from 2014-2015 to 2019-2020, 90 individuals participated in at least four study seasons and 433 doses of HD-IIV3 were administered. The strains included in each vaccine and the study protocol are described in Fig. 1. Table 1 shows demographic and clinical characteristics of the study participants. There were slightly more females (55.6\%), and yearly study enrollment increased over time. There were missing data in our study (i.e., individuals who did not participate in all six influenza seasons), but this did not substantially depart from the missing at random assumption, so multi-level models were used

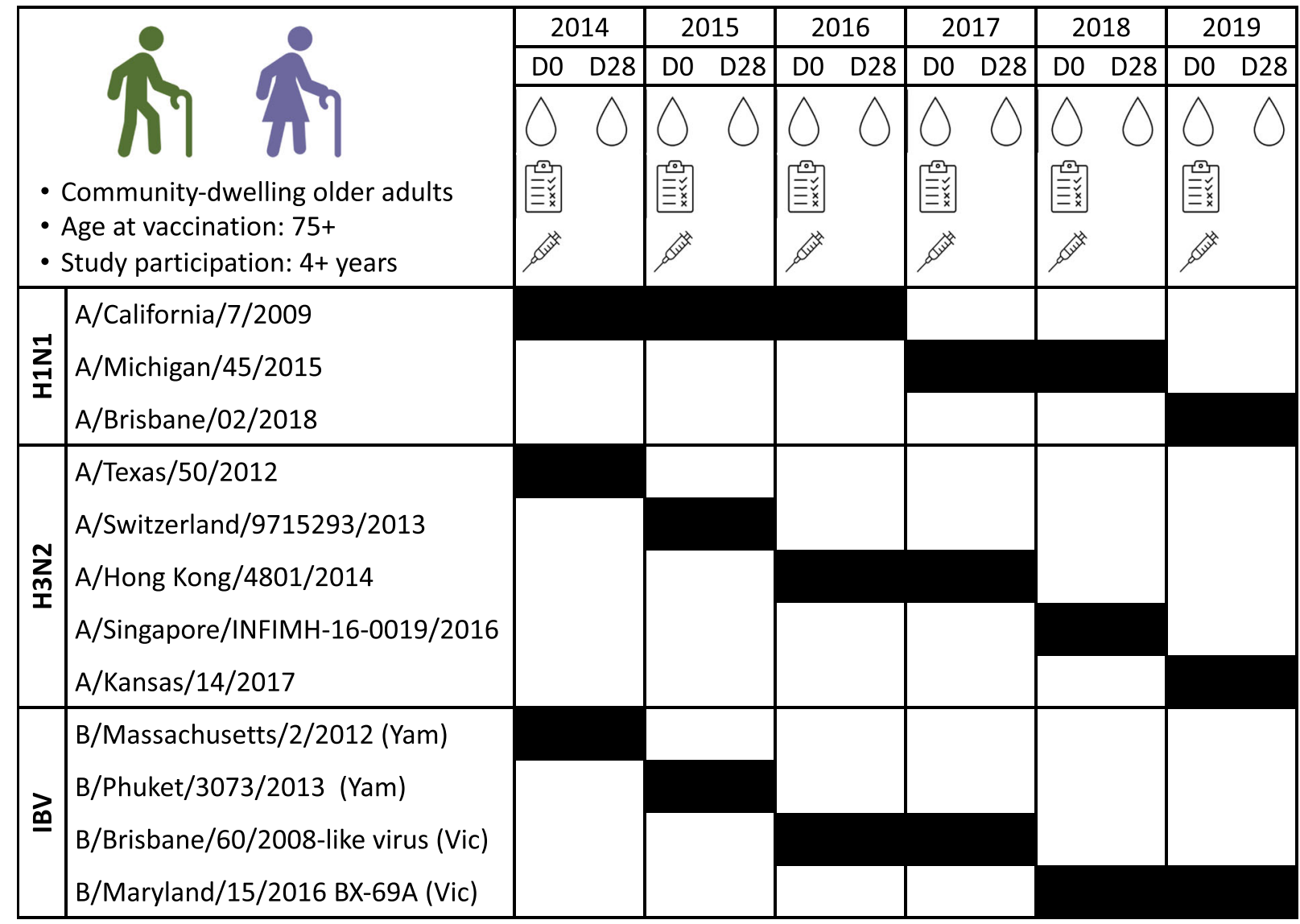
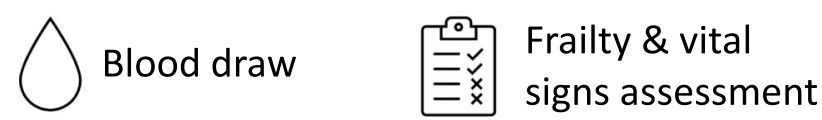

\section{High-dose trivalent inactivated influenza vaccine}

Fig. 1 Study design. Study procedures and the three strains included in each seasonal HD-IIV3 are shown. Serum from blood draws was used to evaluate pre- and post-vaccination strain-specific hemagglutination antibody inhibition (HAl) titers, and frailty was assessed using the Frailty Phenotype. Images were created with BioRender.com. 
Table 1. Summary of study population characteristics.

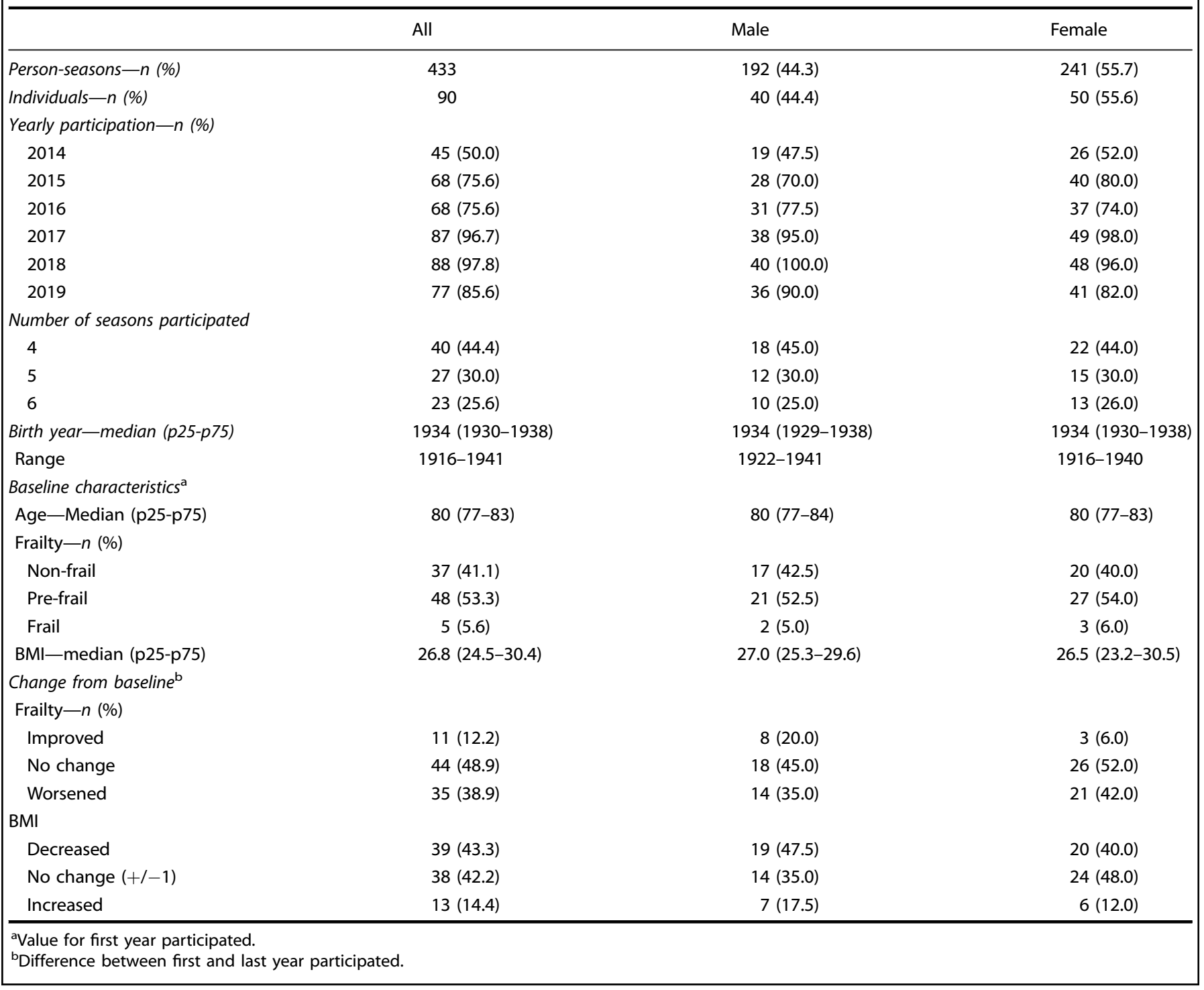

to account for this missingness. Baseline characteristics, measured during the first year of participation, were similar between males and females. The median age at study enrollment was 80 , and $>50 \%$ of participants were classified as pre-frail as per the Fried Frailty Phenotype ${ }^{42}$. Trends in the change of frailty status since baseline were calculated as the difference between the first and last years of participation and differed by sex. A greater proportion of males improved in frailty status, whereas more females either did not change or progressed in frailty status. A greater proportion of males also experienced changes in BMI over the course of the study, while BMI did not change for females.

\section{Pre- and post-vaccination strain-specific HAl titers are high} among repeatedly vaccinated older adults

Pre- and post-vaccination strain-specific HAl titer outcomes to the three HD-IIV3 vaccine strains are summarized and disaggregated by sex in Table 2. Pre- and post-vaccination outcomes stratified by influenza season are detailed in supplement tables (Supplementary Tables 1-6). As expected in this highly vaccinated elderly population, titers and seroprotection rates (defined as an HAl titer $\geq 40^{43-45}$ ) were high. This was particularly true for influenza $B$, where $98 \%$ of the participants were seroprotected prior to immunization.
Post-vaccination, $>94 \%$ of participants achieved seroprotection for $\mathrm{H} 1 \mathrm{~N} 1$ and $\mathrm{H} 3 \mathrm{~N} 2$, while $100 \%$ of participants were seroprotected against influenza B. Because of the lack of variability in postvaccination seroprotection, this outcome was omitted from further analysis. Fold-rise in titers and rates of seroconversion, as defined by $\geq 4$-fold titer increase ${ }^{46}$, were relatively low but were highest for $\mathrm{H} 3 \mathrm{~N} 2$. There were no sex differences in any post-vaccination outcomes. Together, these data indicate that pre-existing and postvaccination strain-specific HAl titers remained high among older adults who were repeatedly vaccinated with HD-IIV3.

Age, sex, BMI, frailty status, and repeated vaccination are not associated with post-vaccination outcomes

We then assessed the relationships between pre-defined host factors (i.e., age, sex, BMI, and frailty status) and post-vaccination strain-specific HAl titer outcomes. When controlling for prevaccination titers and influenza season, neither age, frailty, nor BMI individually had statistically significant associations with post-vaccine titers (Supplementary Fig. $1 \mathrm{~A}-\mathrm{C}$ ), the fold-rise in titers (Fig. 2a-C), or the odds of seroconversion (S Supplementary Fig. 2A-C) for either $\mathrm{H} 1 \mathrm{~N} 1, \mathrm{H} 3 \mathrm{~N} 2$, or influenza B. Inclusion of interaction terms in the models allowed for analysis of sex- 
Table 2. Pre- and post-vaccination hemagglutination antibody inhibition (HAl) titer outcomes.

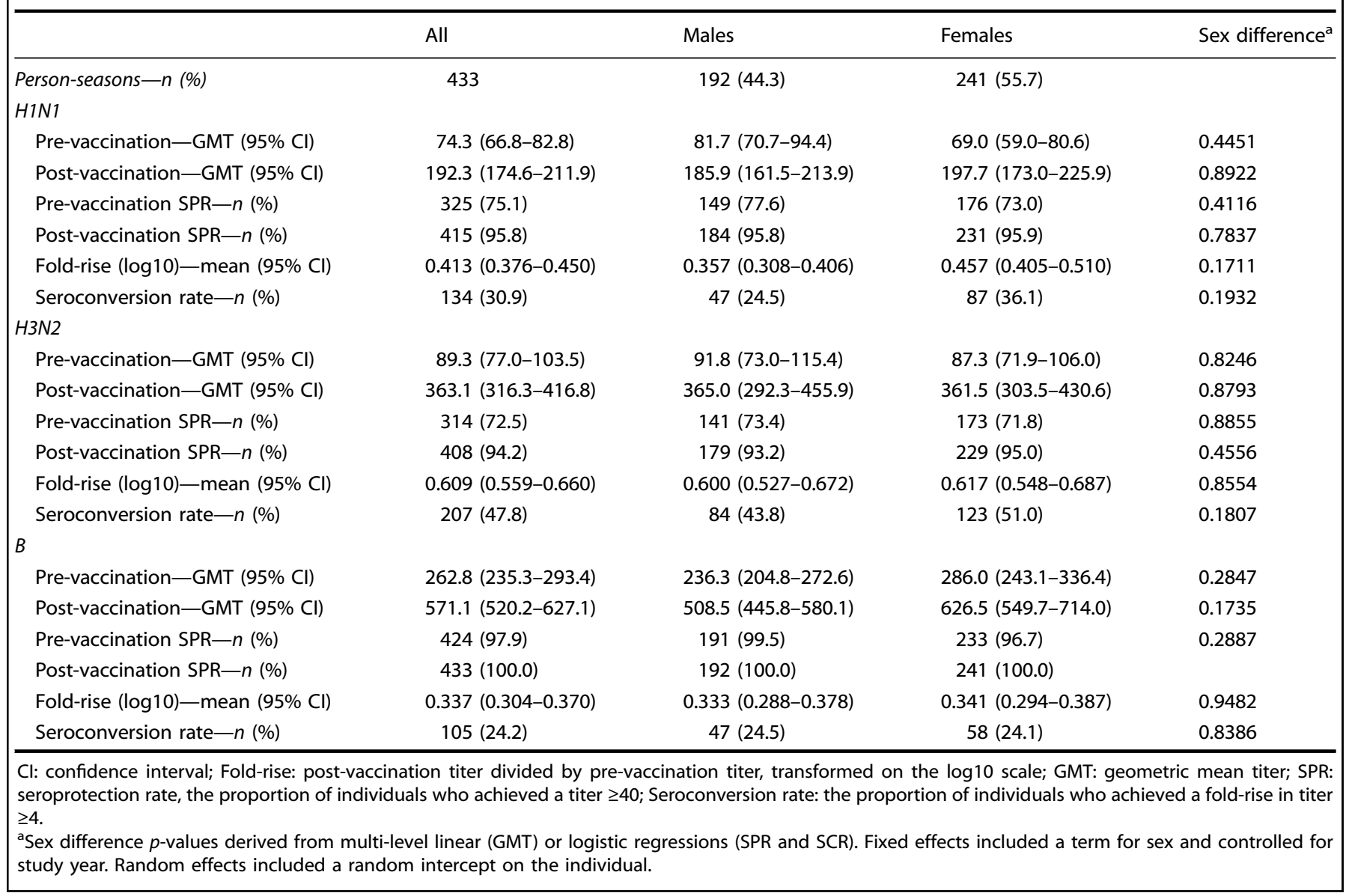

specific contributions of age, frailty, and BMI as well as sex differences in the effects of these host factors. None of the host factors had a statistically significant association with postvaccination titers (Supplementary Fig. $1 \mathrm{~A}-\mathrm{C}$ ), the fold-rise (Fig. $2 \mathrm{a}-\mathrm{C}$ ), or the odds of seroconversion (Supplementary Fig. $2 \mathrm{~A}-\mathrm{C}$ ) for either males or females.

The relationship between the number of years of study participation (measured as a time-varying predictor) and postvaccination HAl titers outcomes was investigated to evaluate potential negative effects of repeated annual vaccination on the humoral immune response to future vaccines. Vaccination status prior to study enrollment could not be verified, but leveraging the longitudinal design of our study, we sought to quantify whether post-vaccination outcomes declined with additional annual vaccination, and whether this effect differed by sex. There was a non-significant negative trend between the number of years participated and the fold-rise in titers (Fig. $2 \mathrm{~d}-\mathrm{g}$ ). These trends were also observed when analyses were repeated using post-vaccination titers (Supplementary Fig. $1 \mathrm{D}-\mathrm{G}$ ) and rates of seroconversion (Supplementary Fig. 2D-G) as outcomes of interest.

\section{Pre-vaccination HAI titers strongly predict post-vaccination outcomes}

Overall, host factors and increasing number of annual vaccinations did not significantly predict any of the post-vaccination antibody titer parameters. Pre-vaccination titers, however, were strong predictors of the fold-rise for $\mathrm{H} 1 \mathrm{~N} 1$ (slope $=-0.15 ; 95 \% \mathrm{Cl}$ : -0.18 ; -0.13 ) (Fig. 2h), H3N2 (slope $=-0.12 ; 95 \% \mathrm{Cl}:-0.15 ;-0.10)$ (Fig. 2i), and influenza $\mathrm{B}$ (slope $=-0.13 ; 95 \% \mathrm{Cl}$ : $-0.15 ;-0.11)($ Fig. $2 \mathrm{j})(p<$
0.0001 for testing the null hypotheses that the slope equals zero for each vaccine strain), such that greater pre-vaccination titers were associated with a smaller fold-rise. Analyses were repeated using post-vaccination titers (Supplementary Fig. $1 \mathrm{H}-\mathrm{K}$ ) and odds of seroconversion (Supplementary Fig. $2 \mathrm{H}-\mathrm{K}$ ) as outcomes of interest, and similarly strong associations were observed with the prevaccination titers. The strength of these associations suggests that post-vaccination outcomes are primarily determined by pre-existing humoral immunity. Thus, in highly vaccinated populations, such as the older adult participants in this study, prevaccination titers are not just confounders to be controlled for in the analysis of post-vaccination humoral immunity but are an outcome of public health importance that illustrate the durability of immunity to influenza from one season to the next. Given the importance of pre-vaccination titers, we focused subsequent analyses on exploring the relationships between host factors and pre-vaccination $\mathrm{HAl}$ titers in the context of advanced age and repeated annual vaccination.

\section{Sex modifies the relationship between age and pre- vaccination HAl titers}

Next, we assessed the relationships between age, frailty, BMI, and pre-vaccination titers (Fig. $3 a-c)$. Neither frailty nor BMI were statistically significantly associated with pre-vaccination HAI titers for all participants or in sex-disaggregated subgroups. Further, there were no statistically significant sex differences in the effects of frailty or BMI on pre-vaccination HAl titers against either H1N1, H3N2, or influenza B. A statistically significant sex by age interaction, however, was observed for H3N2 (Fig. 3b) and for 


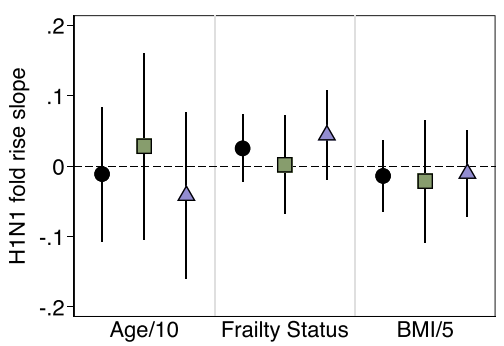

b

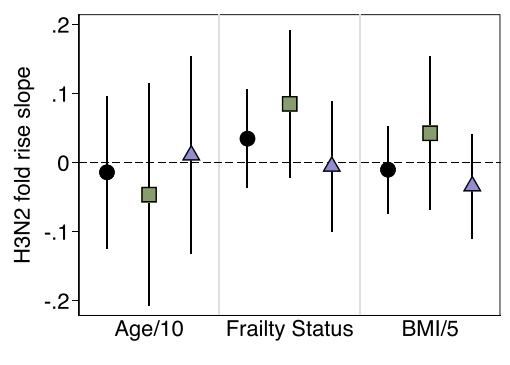

C

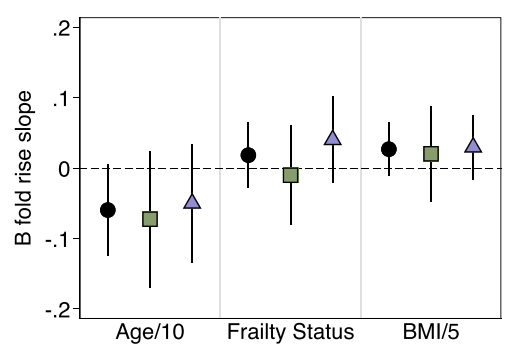

d

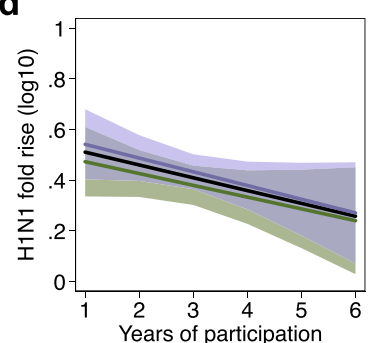

h

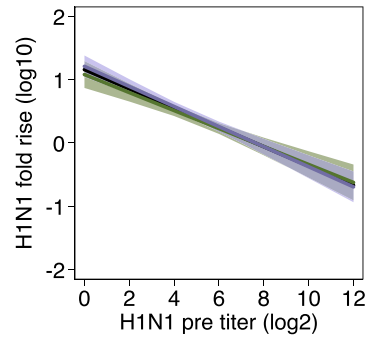

e

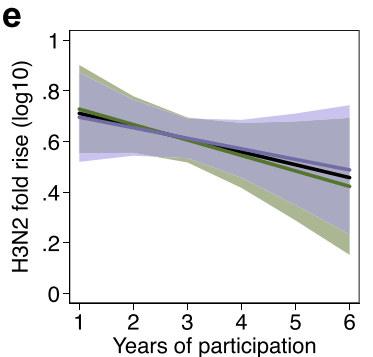

i

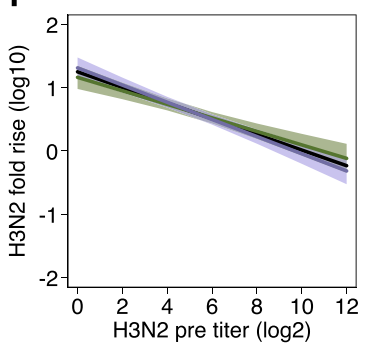

f
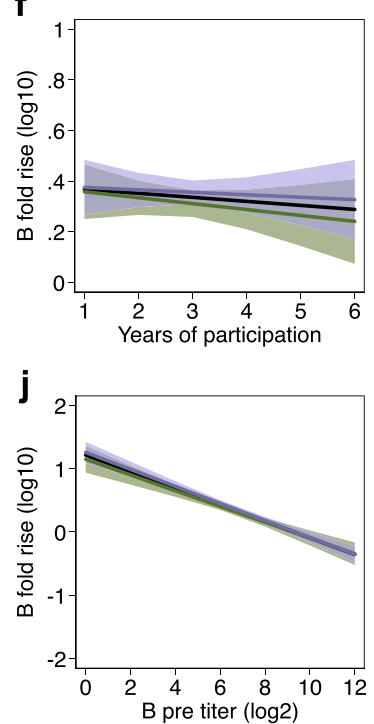

9

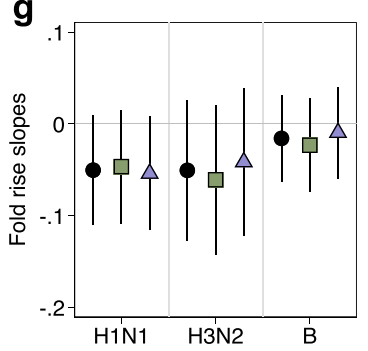

k

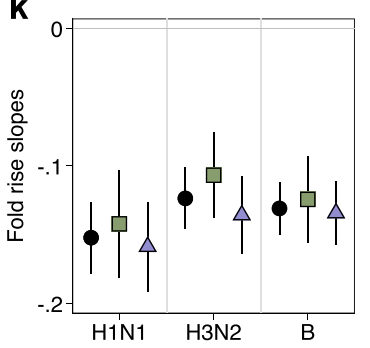

\section{$\triangle$ All $\square$ Male $\triangle$ Female}

Fig. 2 Impact of host factors, repeated vaccination, and pre-vaccination titers on the fold-rise in HAI titers. The relationship of age (in decades, Age/10), frailty status, and BMI (five-unit intervals, BMI/5) with $\log _{10}$-transformed fold-rise in titers (post-titer/pre-titer) are shown as

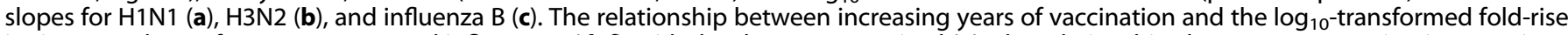
in titers are shown for $\mathrm{H} 1 \mathrm{~N} 1, \mathrm{H} 3 \mathrm{~N} 2$, and influenza $\mathrm{B}(\mathbf{d}-\mathbf{f})$, with the slopes summarized $(\mathbf{g})$. The relationships between pre-vaccination HAI titers and the $\log _{10}$-transformed fold-rise in titers are shown for each vaccine antigen $(\mathbf{h}-\mathbf{j})$, with the slopes summarized (k). Estimates and $95 \%$ confidence intervals were derived from multi-level mixed-effects models with random intercepts on the individual participant. Models controlled for influenza season and pre-vaccination HAl titers (a-g), and either controlled for sex (whole population estimates) or used interaction terms between sex and the host factor of interest to derive sex-specific estimates.

influenza B (Fig. 3c), in which HAl titers declined with age among male but not female participants.

To further interrogate the sex-specific effects of aging, expanded models controlling for frailty and BMI, in addition to influenza season, were constructed. Coefficients from the base (i.e., controlling for infleunza season only) and expanded models are shown in Table 3, and results from expanded models are plotted in Fig. 3d-f. For H1N1, HAl titers tended to increase with age for both males and females, but the increase was not statistically significant (males: 0.49 units per decade, $p=0.152$; females: 0.35 units per decade, $p=0.267$ ), nor was the sex difference in slope $(p=0.676)$ (Fig. $3 d$ ). For H3N2, while titers again tended to increase with age in females $(0.62$ units per decade, $p=0.121)$, they tended to decrease with age in males $(-0.75$ units per decade, $p=0.097)$, leading to a significant sex difference in age slopes (sex by age interaction $=0.137 ; p=$ 0.010) (Fig. 3e). For influenza $B$, titers again tended to increase with age in females ( 0.33 units per decade, $p=0.275$ ) but decreased by 0.78 units per decade in males ( $p=0.023)$ (Fig. 3f).
Like H3N2, the sex difference in the effect of age was significant for influenza B ( $p=0.005)$.

Both the base and expanded models were then amended to include cubic splines to obtain more granular estimates of the effects of age on HAl titers for males and females. Coefficients for base and expanded non-linear models are shown in Table 3, and results from expanded models are plotted in Fig. $3 g-i$. The nonlinear model for $\mathrm{H} 1 \mathrm{~N} 1$ did not differ from the linear model, and no significant effects of age within each sex or difference between the sexes were observed (Fig. $3 \mathrm{~g}$ ). Although the trends in the linear models were similar for $\mathrm{H} 3 \mathrm{~N} 2$ and influenza $\mathrm{B}$, using age as a non-linear predictor revealed that different age categories were driving the overall effects. For $\mathrm{H} 3 \mathrm{~N} 2$, the increase in prevaccination $\mathrm{HAl}$ titers with age in females was driven by individuals in the $80-85$ age category (increase of 0.67 units; $p=0.037$ ), and the decrease in males was driven primarily by people in the 75-80 age category (decrease of 1.78 units; $p=$ 0.012 ) (Fig. 3h). Thus, the sex difference was greatest at the younger end of the cohort ( $p=0.036$ at 75 years of age). Conversely, increasing titers to influenza B with age in females 
a
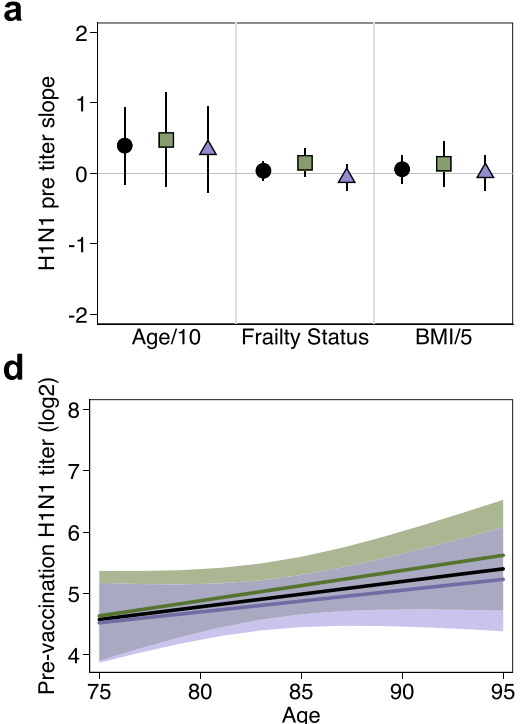

g

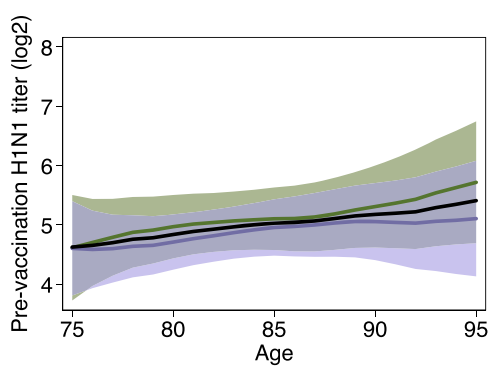

b

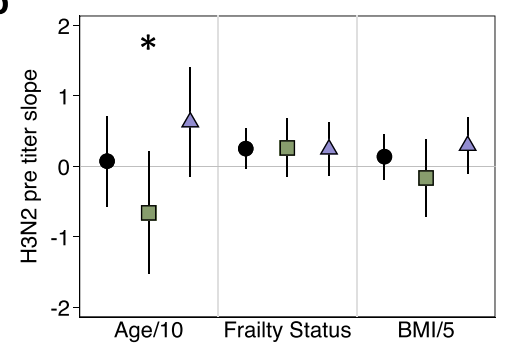

e

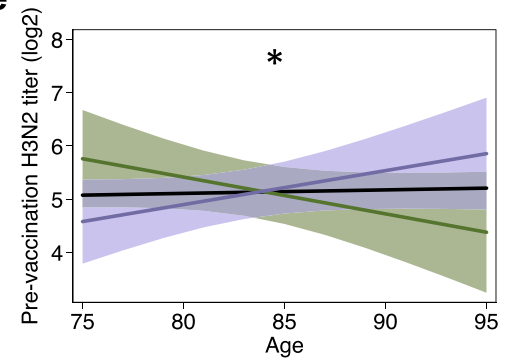

h

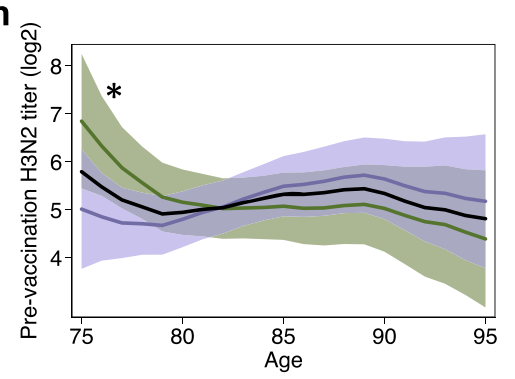

C

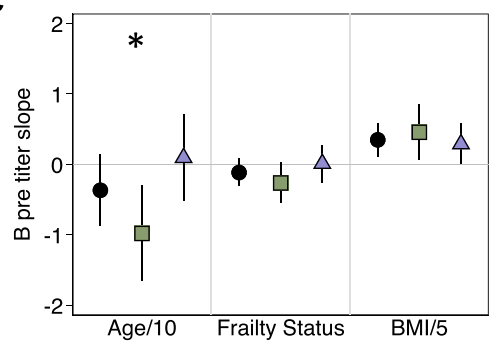

f

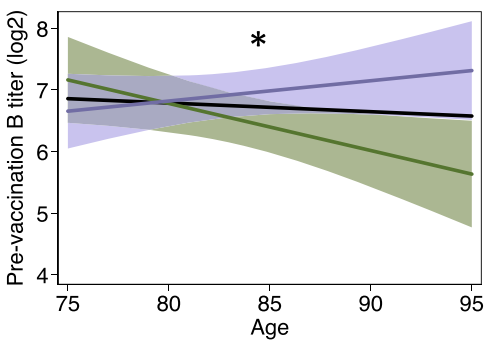

i

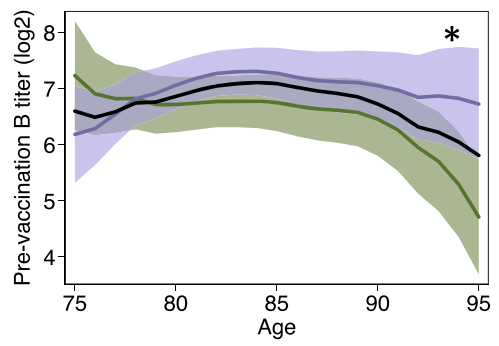

\section{$\square^{\text {All }} \square$ Male $\triangle$ Female}

Fig. 3 Relationship of age, frailty status, and BMI to pre-vaccination hemagglutination antibody inhibition (HAI) titers. Estimates for the relationship of age in decades (Age/10), frailty status, and BMI (five-unit intervals, BMI/5) to pre-vaccination HAl titers were derived from multilevel mixed-effects models controlling for study year for H1N1 (a), H3N2 (b), and influenza B (c). Expanded age models controlling for frailty and BMI are shown for responses to H1N1 (d), H3N2 (e), and influenza B (f). Expanded models for responses to H1N1 (g), H3N2 (h), and influenza B (i) were then amended to include cubic B-splines for age with knots at 5-year intervals. Models for the whole study population adjusted for sex, while sex-specific estimates included an interaction term allowing effects to differ by sex and are shown with $95 \%$ confidence intervals. Asterisks indicate significant sex differences.

were driven by individuals in the 75-80 age category (increase of 1.2 units; $p=0.004)$, whereas for males there was a sharp decline in HAl titers that occurred in participants $90-95$ years of age (decrease of 1.65 units; $p<0.0001$ ) (Fig. 3i). Here, the sex difference in pre-vaccination titers was only significant at the oldest end of the cohort ( $p=0.003$ at 95 years of age). Taken together, these data illustrate sex-specific effects of aging on prevaccination antibody titers to $\mathrm{H} 3 \mathrm{~N} 2$ and influenza $\mathrm{B}$, but not $\mathrm{H} 1 \mathrm{~N} 1$.

To account for the fact that there were several consecutive influenza seasons where the strain included in the vaccine remained constant for either $\mathrm{H} 1 \mathrm{~N} 1, \mathrm{H} 3 \mathrm{~N} 2$, or influenza $B$, the above analyses were repeated controlling for viral vaccine stain rather than influenza season. Reanalasis of prevaccination antibody titers using viral vaccine strain rather than influenza season in the models did not change any of the associations between age and sex described above, and the trend of declining pre-vaccination titers in males to $\mathrm{H} 3 \mathrm{~N} 2$ and influenza B remained (Supplementary Table 7). Further, to illustrate the consequences of ignoring sex as a biological variable, as is commonly encountered in biomedical research ${ }^{47,48}$, analyses were repeated controlling for sex rather than allowing age effects to vary by sex. For H3N2 and influenza B, where the effect of aging was found to be significantly different in males as compared to females, the estimates derived by controlling for sex (black lines in Fig. 3e, $f, h$, i) were not representative of either males or females. In the linear models, for example, controlling for sex led to the incorrect inference that titers remain constant with age, while the interaction models demonstrate that this is false for both males and females. The goodness-of-fit of models controlling for sex and using an age-by-sex interaction term were compared using Akaike's Information Criterion (AIC) in Table 4, where lower values indicate better relative goodness-of-fit. For antibody titers to $\mathrm{H} 3 \mathrm{~N} 2$ and influenza $\mathrm{B}$, despite the penalty for increasing model complexity, fit was improved by including an age-by-sex interaction term that allowed the effect of age to differ by sex. Thus, incorporating sex differences into vaccinology research can lead to more robust analysis.

\section{DISCUSSION}

In this multi-season, longitudinal study of older adults over 75 years of age, pre-vaccination titers, rather than host factors or repeated vaccination, strongly predicted all post-vaccination 
Table 3. Sex-specific effects of age on pre-vaccination hemagglutination antibody inhibition (HAl) titers.

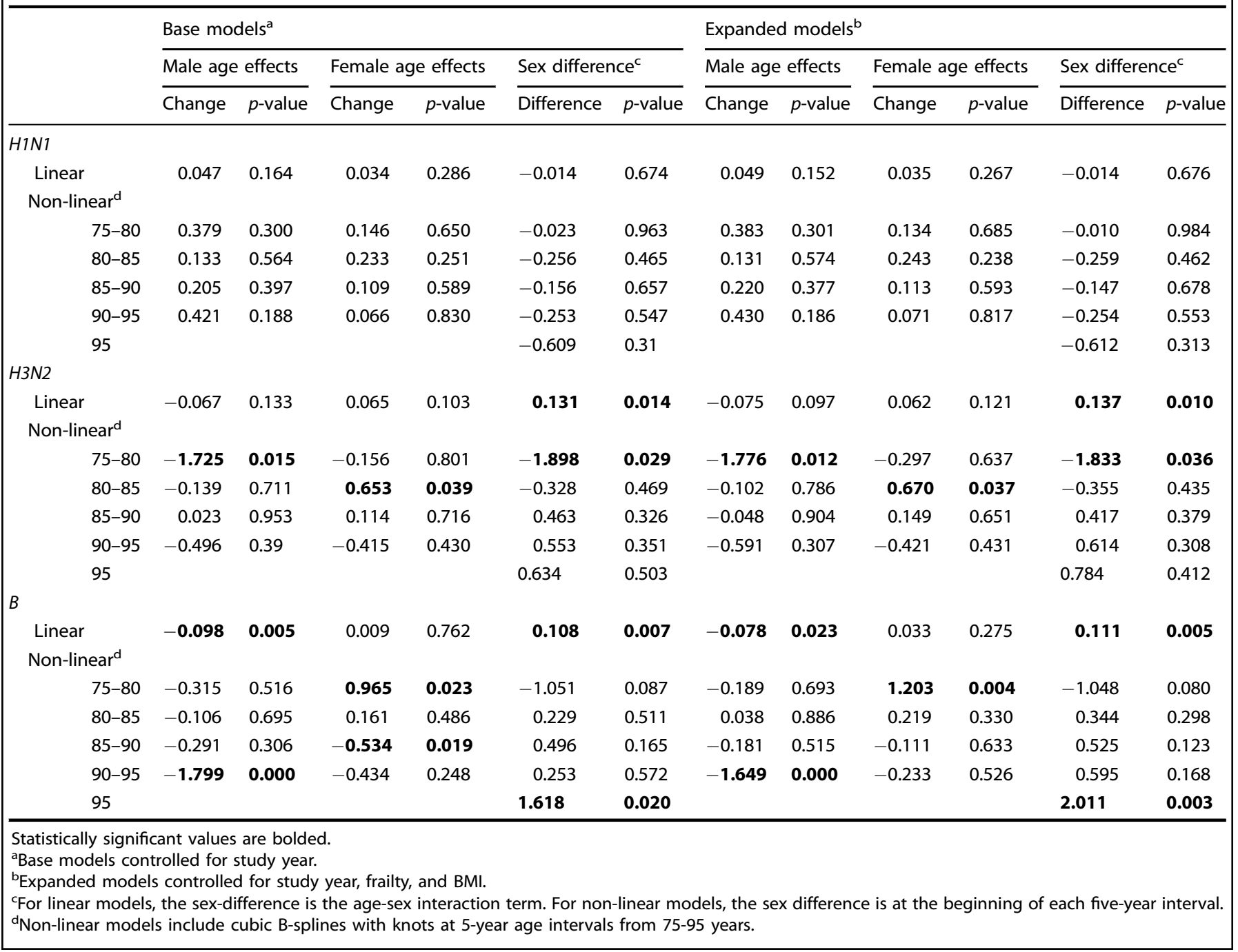

antibody titer outcomes. While it has previously been reported that pre-existing immunity predicts the outcome of vaccination across various age groups ${ }^{15,17}$, the role of sex and age in explaining variability in pre-existing immunity has not been characterized. We report that pre-existing humoral immunity, which reflects the durability of humoral immunity against influenza from previous seasons, displayed an age-by-sex interaction. We found that HAl titers to all three vaccine strains stayed constant in females with age but that HAl titers to H3N2 and influenza B decreased with age in males, leading to significant sex differences in the effect of age for these two vaccine viruses. Thus, sex is a fundamental predictor of the effect of age on pre-existing immunity in this vulnerable population. It has previously been reported that at older ages, there is a malebias in influenza $B$ infection and hospitalization ${ }^{49,50}$. Our results, therefore, provide a potential mechanism for this sex difference and highlight the need to develop better vaccines or vaccination strategies against influenza for older males.

Because older adults are disproportionally burdened by severe disease and mortality from seasonal influenza, significant effort has been devoted to improving annual vaccination coverage for this vulnerable population. Older adults, particularly those who are over 75 years of age, can thus have decades of repeated annual influenza vaccination. Cumulatively, repeated annual vaccination can lead to high pre-vaccination titers, which we observed in this study and previously reported in younger adult healthcare workers, where mandatory vaccination policies result in exceptionally high rates of immunization ${ }^{51}$. Particularly important in older adults, where formation of de novo responses is impaired by immunosenescence ${ }^{52}$, the breadth of pre-existing humoral immunity and the positive predictive value for post-vaccination titers can thus be harnessed to elicit protection ${ }^{27}$. The clinical and scientific implications of this notion are far-reaching and longterm, as the Advisory Committee on Immunization Practices (ACIP) of the CDC has recommended annual influenza vaccination for anyone aged 6 months and older since $2010^{53}$.

For many vaccines, antibody titers wane over time ${ }^{54-57}$. Influenza vaccines are unique in this respect due to the recommendation for yearly immunization and exceptional antigenic diversity, which alter the dynamics of waning immunity. The constant pre-vaccination HAl titers with age in females seen in our study suggest that females benefit from a booster effect from each successive annual vaccination that appears to prevent antibody waning. This influenza-specific effect has been reported elsewhere, where samples collected from individuals over a 20 year period revealed longitudinal increases in neutralizing titers to influenza ${ }^{58}$. However, our data suggest that this effect is absent in males for $\mathrm{H} 3 \mathrm{~N} 2$ and influenza $\mathrm{B}$. The reasons for this sex difference are unknown, but may be attributable to the compounding effects of females developing stronger responses to influenza infection 
Table 4. Goodness-of-fit comparison of pre-vaccination age models.

\begin{tabular}{|c|c|c|c|c|}
\hline \multirow{3}{*}{$\begin{array}{l}\text { Age-sex interaction } \\
\text { H1N1 }\end{array}$} & \multicolumn{2}{|c|}{ Base models ${ }^{a}$} & \multicolumn{2}{|c|}{ Expanded models ${ }^{b}$} \\
\hline & - & + & - & + \\
\hline & & & & \\
\hline Linear age & 1138.86 & 1140.69 & 1131.05 & 1132.87 \\
\hline Non-linear age & 1144.47 & 1151.51 & 1136.73 & 1143.72 \\
\hline \multicolumn{5}{|l|}{ H3N2 } \\
\hline Linear age & 1693.38 & 1689.57 & 1666.94 & 1662.83 \\
\hline Non-linear age & 1694.58 & 1694.83 & 1667.28 & 1667.95 \\
\hline \multicolumn{5}{|l|}{ B } \\
\hline Linear age & 1398.31 & 1393.17 & 1355.04 & 1349.28 \\
\hline Non-linear age & 1388.09 & 1378.85 & 1344.94 & 1337.26 \\
\hline $\begin{array}{l}\text { The lowest AIC, } \\
\text { each virus. } \\
\text { aBase models cont } \\
\text { bExpanded model }\end{array}$ & o & the $b$ & hodel, & Ided for \\
\hline
\end{tabular}

and vaccination throughout adulthood ${ }^{59}$, leading to a more robust repertoire of memory B cells that recognize conserved epitopes on drifted virus strains. It is speculated that in older adults, consistently inferior responses among males may manifest as a lack of memory B cells that can be boosted by drifted viruses to counteract waning of antibody over time, thus resulting in decreasing pre-vaccination titers with age.

Notably the sex difference was absent for responses to H1N1 vaccine antigens. A possible explanation for this lies in the differing evolutionary rates of the three vaccine viruses. H1N1 viruses experience slower evolution than $\mathrm{H} 3 \mathrm{~N} 2$ viruses $^{60}$ and the influenza B/Victoria lineage ${ }^{61}$. In addition, the global co-circulation of B/Yamagata and B/Victoria lineages leads to increased exposure to divergent antigens ${ }^{62}$. Accordingly, over the six influenza vaccine seasons included in our study, and in the past decade, vaccine antigens were significantly more variable for $\mathrm{H} 3 \mathrm{~N} 2$ and influenza $B$ than for $\mathrm{H} 1 \mathrm{~N} 1^{63}$. It is thus possible that repeated exposure to the same H1N1 strain sufficiently boosted male steady-state immunity to mask sex differences in the immune response. Conversely, for $\mathrm{H} 3 \mathrm{~N} 2$ and influenza $\mathrm{B}$, sequential exposure to drifted viruses required robust and broad responses to allow for boosting of steady-state immunity, which may have only been present in females. Another possible explanation is immunological imprinting in youth, as it has a lifelong impact on subsequent immune responses to influenza infection and vaccination ${ }^{23,64}$. Individuals in our cohort, born from 1916-1941, may have been exposed to H1N1 in their youth, while the 1918 pandemic virus continued to circulate, but were likely exposed to H3N2 and influenza B later in life ${ }^{65}$. It is, therefore, possible that strong immune imprinting to $\mathrm{H} 1 \mathrm{~N} 1$ virus strains masked the sex differences otherwise observed for $\mathrm{H} 3 \mathrm{~N} 2$ and influenza $\mathrm{B}$.

Our study had several strengths and limitations. First, this was an observational study that was not specifically designed to interrogate sex differences in the immune response to influenza vaccination. To overcome small yearly sample sizes, six influenza seasons were pooled together, and statistical methods were used to control for annual variation in vaccine virus strains and repeated measurements on participants. The resulting multiseason nature of this work improves generalizability to future influenza seasons. Secondly, the humoral immune response to vaccination was strain-specific HAI titers, which are the standard in the field, but lack the functional quality of microneutralization assays $^{66}$. Relying solely on serological samples also prohibited mechanistic investigation at the cellular level. In-depth studies of cellular and transcriptional mechanisms underlying the sex differences observed in this cohort are on-going. Third, the lack of racial diversity in our cohort must be noted, as it prohibited us from investigating race as a host factor of interest, which should be considered in future studies. Although the study lacked racial diversity, the cohort was diverse in terms of age at vaccination, allowing us to study effects in the 'oldest' old subset. In addition, we were unable to ascertain vaccination history for the participants prior to enrollment in the study. Previous research suggests that influenza vaccination coverage is similar among older men and women ${ }^{67}$, such that it is unlikely that gender differences in vaccination history confounded the results observed. Finally, a major strength of this study is the intersectional approach to analysis, which allowed for interrogation of effects both between and within groups (i.e., between and among males and females), leading to a richer and more nuanced interpretation $^{68}$.

In conclusion, we demonstrate that in highly vaccinated older adults, pre-vaccination HAl titers, rather than age, sex, BMI, frailty, or repeated vaccination, predict post-vaccination parameters of humoral immunity. These pre-vaccination titers change with age in a sex-specific manner, such that older males are particularly vulnerable to lower levels of pre-existing humoral immunity. These findings provide a basis for future studies to investigate the predictive value of host factors and vaccination history in protection from influenza, which could ultimately be a valuable tool in a clinical setting. Further research should focus on elucidating the mechanisms underlying this sex difference, as well as novel vaccination strategies to harness the breadth of preexisting immunity in older adults to provide better protection against influenza for this vulnerable population.

\section{METHODS}

\section{Study population and protocol}

During the 2014-2015 to 2019-2020 influenza seasons, we enrolled community-dwelling older adults above 75 years of age who had not yet received a seasonal influenza vaccine. Individuals who had a history of allergic reaction to influenza vaccines or to eggs, were currently taking oral steroids, or had worsening or new-onset of immune-modulating conditions (e.g., rheumatoid arthritis, hematologic malignancies, etc) were excluded. Study participants came to the Clinical Research Unit at Johns Hopkins Institute of Clinical and Translational Research on the Johns Hopkins Bayview Medical Center campus, or study visits were conducted at participants' home as needed. A detailed medical history was obtained, vital signs were measured and frailty was assessed as per the Fried Frailty Phenotype ${ }^{42}$. After a pre-vaccination blood draw, participants received HDIIV3 (Fluzone ${ }^{\circledR}$ High-Dose, Sanofi Pasteur, PA, USA). A second blood sample was collected between 21 and 28 days after vaccine administration (Fig. 1). To focus on the context of repeated annual vaccination, only individuals who participated in a minimum of 4 influenza seasons were included in this analysis.

\section{Ethics}

Written, informed consent was obtained from all participants. The study protocol was approved by the Johns Hopkins School of Medicine Institutional Review Board. The study is registered on clinicaltrials.gov (NCT02200276).

\section{Hemagglutination inhibition assays}

Validated HAl assays were performed by Sanofi Pasteur and used to quantify antibody titers against the three influenza virus strains ( $\mathrm{H} 1 \mathrm{~N} 1, \mathrm{H} 3 \mathrm{~N} 2$, and $\mathrm{B})$ included in each season's vaccine ${ }^{69}$. Briefly, serum was incubated with type III neuraminidase to eliminate non-specific inhibitors and then with turkey red blood cells to adsorb non-specific agglutinins. Two-fold serial dilutions of sera, beginning at a 1:10 dilution, were then performed in duplicate, and sera were incubated with influenza virus ( 4 hemagglutination units/25 $\mu \mathrm{l}$ ). Turkey red blood cells were then added, and the titer defined as the highest dilution in which hemagglutination of turkey red blood cells was inhibited. 


\section{Definitions and categorization of predictor variables}

Sex was used as a dichotomous variable based on self-report. Age was calculated based on the date of vaccination and used as a continuous variable. The frailty assessment was based on the presence or absence of five measurable characteristics: slowed motor performance (by walking speed), poor endurance and energy (by self-report of exhaustion), weakness (by grip strength), shrinking (by unintentional weight loss), and low physical activity (by self-report) ${ }^{34,42}$. Participants with three or more out of these five characteristics were defined as frail, those with one or two as prefrail, and those with none as non-frail. BMI was calculated based on measured height and weight and used as a continuous variable. Influenza season (i.e., 2014-2015 to 2019-2020) was used as categorical variable so as not to imply a linear relationship from yearto-year. The influenza season was included as a dummy variable in analyses to account for possible confounding due to variation in the vaccine composition over time. In sensitivity analyses, viral strain was used as an alternative approach to control for confounding due to antigenic drift. Number of years of study participation was defined as the number of vaccines administered to an individual as part of the study at the time that the outcome was measured each year. Number of years of study participation was used as a time-varying continuous predictor ranging from 1 to 6 (i.e. set to one the first year an individual participated, two the second year an individual participated, etc...).

\section{Outcome variables}

Geometric mean titers were calculated both pre- and post-vaccination. For regression analysis, titers were transformed to a $\log _{2}$ scale to achieve an approximately normal distribution. The fold-rise in titer was calculated as post-vaccination titers divided by pre-vaccination titers, and $\log _{10}$ transformed to achieve a normal distribution. Seroconversion was defined as achieving a fold-rise $\geq 4$ and used as a binary outcome. Seroprotection was defined as a titer $\geq 40$ and used as a binary outcome.

\section{Statistical analysis}

To account for repeated measures on participants, multi-level mixedeffects models with random intercepts on the individual were used. Although there was missing data in our study, we do not anticipate substantial departure from the missing at random assumption. The mixedeffects method was selected because it is considered to be robust in addressing "non-informative" missing data ${ }^{70}$. Following standard risk factor analysis procedure, the contributions of host factors of interest were first assessed individually. Based on the a priori hypotheses of this analysis, fixed effects of the base models for post-vaccination outcomes adjusted for influenza season, pre-vaccination titers and included interaction terms to allow the effect of the host factor to differ for males and females. Fixed effects of the base models for pre-vaccination titers adjusted for influenza season and included interaction terms to allow effects to differ by sex. Where significant sex differences were found, further analysis controlled for additional covariates, and used cubic B-splines to investigate non-linear relationships ${ }^{71}$. The relative goodness-of-fit of various models were compared using Akaike's Information Criterion. For graphs, predictions were capped at 95 years of age due to low sample size and large uncertainty in estimates above 95 years. Coefficients were considered statistically significant if $95 \%$ confidence intervals did not span the null value of zero (i.e., $p<0.05$ ). Analysis was performed in Stata 15 (StataCorp).

\section{Reporting summary}

Further information on research design is available in the Nature Research Reporting Summary linked to this article.

\section{DATA AVAILABILITY}

The data that support the findings of this study are available from the corresponding author upon request.

Received: 21 July 2021; Accepted: 2 November 2021; Published online: 09 December 2021

\section{REFERENCES}

1. Macias, A. E. et al. The disease burden of influenza beyond respiratory illness. Vaccine https://doi.org/10.1016/j.vaccine.2020.09.048 (2020).
2. Iuliano, A. D. et al. Estimates of global seasonal influenza-associated respiratory mortality: a modelling study. Lancet 391, 1285-1300 (2018).

3. Global Burden of Disease Influenza Collaborators. et al. Mortality, morbidity, and hospitalisations due to influenza lower respiratory tract infections, 2017: an analysis for the Global Burden of Disease Study 2017. Lancet Respir. Med. 7, 69-89 (2018). PMID - 30553848.

4. Gnanasekaran, G., Biedenbender, R., Davidson, H. E. \& Gravenstein, S. Vaccinations for the Older Adult. Clin. Geriatr. Med 32, 609-625 (2016).

5. Hamborsky, J., Kroger, A. \& Wolfe, S. Epidemiology and prevention of vaccinepreventable diseases. (US Department of Health \& Human Services, Centers for Disease Control and Prevention 2015).

6. Grohskopf, L. A. et al. Prevention and control of seasonal influenza with vaccines: recommendations of the Advisory Committee on Immunization Practices-United States, 2020-21 influenza season. MMWR Recomm. Rep. 69, 1 (2020).

7. DiazGranados, C. A. et al. Efficacy of high-dose versus standard-dose influenza vaccine in older adults. N. Engl. J. Med. 371, 635-645 (2014).

8. Centers for Disease Control and Prevention. Flu Vaccination Coverage, United States, 2019-20 Influenza Season, https://www.cdc.gov/flu/fluvaxview/coverage1920estimates.htm (2020).

9. Crooke, S. N., Ovsyannikova, I. G., Poland, G. A. \& Kennedy, R. B. Immunosenescence: a systems-level overview of immune cell biology and strategies for improving vaccine responses. Exp. Gerontol. 124, 110632 (2019).

10. Weinberger, B. Vaccines for the elderly: current use and future challenges. Immun. Ageing15, 3 (2018).

11. Chen, Y., Liu, S. \& Leng, S. X. Chronic low-grade inflammatory phenotype (CLIP) and senescent immune dysregulation. Clin. Ther. 41, 400-409 (2019).

12. Demicheli, V., Jefferson, T., Ferroni, E., Rivetti, A. \& Di Pietrantonj, C. Vaccines for preventing influenza in healthy adults. Cochrane Database Syst. Rev. https://doi. org/10.1002/14651858.CD001269.pub6 (2018).

13. Nichols, M. K. et al. Influenza vaccine effectiveness to prevent influenza-related hospitalizations and serious outcomes in Canadian adults over the 2011/12 through 2013/14 influenza seasons: a pooled analysis from the Canadian Immunization Research Network (CIRN) Serious Outcomes Surveillance (SOS Network). Vaccine 36, 2166-2175 (2018).

14. Rondy, M. et al. Effectiveness of influenza vaccines in preventing severe influenza illness among adults: a systematic review and meta-analysis of test-negative design case-control studies. J. Infect. 75, 381-394 (2017).

15. Kwong, J. C. et al. The impact of repeated vaccination using 10-year vaccination history on protection against influenza in older adults: a test-negative design study across the 2010/11 to 2015/16 influenza seasons in Ontario, Canada. Eurosurveillance 25, 1900245 (2020).

16. McLean, H. Q. et al. Impact of repeated vaccination on vaccine effectiveness against influenza $\mathrm{A}(\mathrm{H} 3 \mathrm{~N} 2)$ and $\mathrm{B}$ during 8 seasons. Clin. Infect. Dis. 59, 1375-1385 (2014).

17. Sung, M.-H., Shen, Y., Handel, A., Bahl, J. \& Ross, T. M. Longitudinal assessment of immune responses to repeated annual Influenza vaccination in a human cohort of adults and teenagers. Front. Immunol. 12, 472 (2021).

18. Belongia, E. A. et al. Repeated annual influenza vaccination and vaccine effectiveness: review of evidence. Expert Rev. Vaccines 16, 723-736 (2017).

19. Bartoszko, J. J. et al. Does consecutive influenza vaccination reduce protection against influenza: a systematic review and meta-analysis. Vaccine 36, 3434-3444 (2018).

20. Cheng, A. C. et al. Repeated vaccination does not appear to impact upon influenza vaccine effectiveness against Hospitalization with confirmed influenza. Clin. Infect. Dis. 64, 1564-1572 (2017).

21. Casado, I. et al. Repeated influenza vaccination for preventing severe and fatal influenza infection in older adults: a multicentre case-control study. Can. Med. Assoc. J. 190, E3-E12 (2018).

22. Örtqvist, Å., Brytting, M., Leval, A. \& Hergens, M.-P. Impact of repeated influenza vaccinations in persons over 65 years of age: A large population-based cohort study of severe influenza over six consecutive seasons, 2011/12-2016/17. Vaccine 36, 5556-5564 (2018). PMID - 30076104.

23. Jang, H. \& Ross, T. M. Preexisting influenza specific immunity and vaccine effectiveness. Expert Rev. Vaccines 18, 1-9 (2019). PMID - 31575308.

24. Henry, C., Palm, A.-K. E., Krammer, F. \& Wilson, P. C. From original antigenic sin to the universal influenza virus vaccine. Trends Immunol. 39, 70-79 (2018). PMID 28867526.

25. Linderman, S. L. et al. Influenza Immunization in the Context of Preexisting Immunity. Cold Spring Harb. Perspect. Med. a040964, https://doi.org/10.1101/ cshperspect.a040964 (2020).

26. Guthmiller, J. J., Utset, H. A. \& Wilson, P. C. B Cell responses against influenza viruses: short-lived humoral immunity against a life-long threat. Vaccine 13, 965 (2021).

27. Guthmiller, J. J. \& Wilson, P. C. Harnessing immune history to combat influenza viruses. Curr. Opin. Immunol. 53, 187-195 (2018).

28. Ellebedy, A. H. Immunizing the immune: can we overcome influenza's most formidable challenge? Vaccines (Basel). 6, 68 (2018). 
29. Falsey, A. R., Treanor, J. J., Tornieporth, N., Capellan, J. \& Gorse, G. J. Randomized, double-blind controlled phase 3 trial comparing the immunogenicity of highdose and standard-dose influenza vaccine in adults 65 years of age and older. $J$. Infect. Dis. 200, 172-180 (2009).

30. Potluri, T. et al. Age-associated changes in the impact of sex steroids on influenza vaccine responses in males and females. Npj Vaccines 4, 29 (2019).

31. Ciarambino, T., Para, O. \& Giordano, M. Immune system and COVID-19 by sex differences and age. Women's Health 17, 17455065211022262 (2021).

32. Meester, l. et al. SeXY chromosomes and the immune system: reflections after a comparative study. Biol. Sex. Differ. 11, 1-13 (2020).

33. Chen, Y. et al. Aging in COVID-19: vulnerability, immunity and intervention. Ageing Res. Rev 65, 101205 (2020).

34. Yao, X. et al. Frailty is associated with impairment of vaccine-induced antibody response and increase in post-vaccination influenza infection in communitydwelling older adults. Vaccine 29, 5015-5021 (2011).

35. Narang, V. et al. Influenza vaccine-induced antibody responses are not impaired by frailty in the community-dwelling elderly with natural influenza exposure. Front. Immunol. 9, 2465 (2018).

36. Epps, P. V. et al. Preexisting immunity, not frailty phenotype, predicts influenza postvaccination titers among older veterans. Clin. Vaccin. Immunol. 24, e00498-00416 (2017).

37. Bauer, J. M. et al. Influenza vaccine response in community-dwelling German prefrail and frail individuals. Immun. Ageing 14, 17 (2017). PMID - 28694834.

38. Moehling, K. K. et al. The effect of frailty on HAI response to influenza vaccine among community-dwelling adults $\geq 50$ years of age. Hum. Vacc Immunother. 14, 361-367 (2017).

39. Moehling, K. K. et al. The impact of physical frailty on the response to inactivated influenza vaccine in older adults. Aging 12 https://doi.org/10.18632/aging.202207 (2020).

40. Loeb, N. et al. Frailty is associated with increased hemagglutination-inhibition titres in a 4-year randomized trial comparing standard and high dose influenza vaccination. Open Forum Infect. Dis. 7, ofaa148 (2020).

41. Frasca, D. et al. Obesity decreases B cell responses in young and elderly individuals. Obesity 24, 615-625 (2016).

42. Fried, L. P. et al. Frailty in older adults: evidence for a phenotype. J. Gerontol. Ser. Biol. Sci. Med. Sci. 56, M146-M157 (2001). PMID - 11253156.

43. Ward, B. J. et al. The establishment of surrogates and correlates of protection: Useful tools for the licensure of effective influenza vaccines? Hum. Vacc Immunother. 14, 647-656 (2018).

44. Al-Khayatt, R., Jennings, R. \& Potter, C. Interpretation of responses and protective levels of antibody against attenuated influenza $A$ viruses using single radial haemolysis. Epidemiol. Infect. 93, 301-312 (1984).

45. Hobson, D., Curry, R., Beare, A. \& Ward-Gardner, A. The role of serum haemagglutination-inhibiting antibody in protection against challenge infection with influenza A2 and B viruses. Epidemiol. Infect. 70, 767-777 (1972).

46. Reber, A. \& Katz, J. Immunological assessment of influenza vaccines and immune correlates of protection. Expert Rev. Vaccines 12, 519-536 (2013).

47. Shapiro, J. R., Klein, S. L. \& Morgan, R. Stop 'controlling' for sex and gender in global health research. BMJ Glob. Heal 6, e005714 (2021).

48. Klein, S. L., Jedlicka, A. \& Pekosz, A. The Xs and $Y$ of immune responses to viral vaccines. Lancet Infect. Dis. 10, 338-349 (2010).

49. Wong, K. C., Luscombe, G. M. \& Hawke, C. Influenza infections in Australia 2009-2015: is there a combined effect of age and sex on susceptibility to virus subtypes. BMC Infect. Dis. 19, 42 (2019).

50. Wang, X.-L. et al. Age and sex differences in rates of influenza-associated hospitalizations in Hong Kong. Am. J. Epidemiol. 182, 335-344 (2015).

51. Kuo, H. et al. Sex-specific effects of age and body mass index on antibody responses to seasonal influenza vaccines in healthcare workers. Vaccine https:// doi.org/10.1016/j.vaccine.2021.02.047 (2021).

52. Weinberger, B., Herndler-Brandstetter, D., Schwanninger, A., Weiskopf, D. \& Grubeck-Loebenstein, B. Biology of immune responses to vaccines in elderly persons. Clin. Infect. Dis. 46, 1078-1084 (2008).

53. Centers for Disease Control and Prevention. Influenza Historic Timeline, https://www. cdc.gov/flu/pandemic-resources/pandemic-timeline-1930-and-beyond.htm (2019).

54. Bitzegeio, J., Majowicz, S., Matysiak-Klose, D., Sagebiel, D. \& Werber, D. Estimating age-specific vaccine effectiveness using data from a large measles outbreak in Berlin, Germany, 2014/15: evidence for waning immunity. Eurosurveillance 24, 1800529 (2019)

55. Crooke, S. N. et al. Durability of humoral immune responses to rubella following MMR vaccination. Vaccine 38, 8185-8193 (2020).

56. Savage, R. D. et al. A multisite study of pertussis vaccine effectiveness by time since last vaccine dose from three Canadian provinces: A Canadian Immunization Research Network study. Vaccine 39, 2772-2779 (2021).
57. Posuwan, N. et al. The success of a universal hepatitis B immunization program as part of Thailand's EPI after 22 years' implementation. PLOS ONE 11, e0150499 (2016).

58. Miller, M. S. et al. Neutralizing antibodies against previously encountered influenza virus strains increase over time: a longitudinal analysis. Sci. Transl. Med 5, 198ra107 (2013)

59. Flanagan, K. L., Fink, A. L., Plebanski, M. \& Klein, S. L. Sex and gender differences in the outcomes of vaccination over the life course. Annu Rev. Cell Dev. Bi 33, 577-599 (2017)

60. Liu, M. et al. Antigenic patterns and evolution of the human influenza $A(H 1 N 1)$ virus. Sci. Rep. 5, 1-8 (2015).

61. Vijaykrishna, D. et al. The contrasting phylodynamics of human influenza B viruses. Elife 4, e05055 (2015).

62. Caini, S. et al. The epidemiological signature of influenza $B$ virus and its B/Victoria and B/Yamagata lineages in the 21st century. PLoS ONE 14, e0222381 (2019).

63. World Health Organization. WHO recommendations on the composition of influenza virus vaccines, https://www.who.int/influenza/vaccines/virus/recommendations/en/ (2021).

64. Auladell, M. et al. Recalling the future: immunological memory toward unpredictable influenza viruses. Front. Immunol. 10, 1400 (2019). PMID - 31312199.

65. Hannoun, C. The evolving history of influenza viruses and influenza vaccines. Expert. Rev. Vaccines 12, 1085-1094 (2013).

66. Dunning, A. J. et al. Correlates of protection against influenza in the elderly: results from an influenza vaccine efficacy trial. Clin. Vaccin. Immunol. 23, 228-235 (2016).

67. Roy, M., Sherrard, L., Dubé, È. \& Gilbert, N. L. Determinants of non-vaccination against seasonal influenza. Health Rep. 29, 12-22 (2018).

68. Shapiro, J. R., Klein, S. L. \& Morgan, R. COVID-19: use intersectional analyses to close gaps in outcomes and vaccination. Nature 591, 202 (2021).

69. Greenberg, D. P. et al. Safety and immunogenicity of a quadrivalent inactivated influenza vaccine compared to licensed trivalent inactivated influenza vaccines in adults. Vaccine 31, 770-776 (2013).

70. Schafer, J. L. \& Graham, J. W. Missing data: our view of the state of the art. Psychol. Methods 7, 147 (2002).

71. Newson, R. B. Sensible parameters for univariate and multivariate splines. Stata J. 12, 479-504 (2012).

\section{ACKNOWLEDGEMENTS}

The authors thank the participants, as well as the clinical staff at the Healthy Aging Studies Unit and the Johns Hopkins University Institute for Clinical and Translational Research. The authors would also like to than Dr. Andrew Pekosz for helpful discussion and feedback. Sanofi Pasteur provided HD-IIV3 and performed HAI testing, but remained blinded to the results and had no further scientific input. This work was supported in part by National Institute of Health $(\mathrm{NIH}) /$ National Institute of Allergy and Infectious Diseases R01 Al108907 to S.X.L., NIH/National Institute on Aging Specialized Center of Research Excellence U54 AG062333 awarded to S.L.K. and funding from Irma and Paul Milstein Program for Senior Health, Milstein Medical Asian American Partnership (MMAAP) Foundation of USA to S.X.L., J.R.S was supported by a training award from the Fonds de recherche du Québec - Santé (File \#287609). C.J.W, X.X.N. and S.F.L. were supported by Irma and Paul Milstein Program for Senior Health fellowship awards from MMAAP Foundation of USA (www. mmaapf.org).

\section{AUTHOR CONTRIBUTIONS}

S.X.L. designed and oversaw the study. E.A., D.C.K., and E.S-M collected data and biological samples. H.F.L., Y.Y.C., Y.C., C.J.W., X.X.N., L.W., and S.F.L. processed biological samples. J.R.S., K.M., and R.S. entered and cleaned data. J.R.S. conceived of the analytical approach and experimental questions and performed analyses under the mentorship of S.L.Z., R.M., and S.L.K., K.M., H.K., and P.S. contributed to analyses. J.R.S. wrote the manuscript, with significant editorial contributions and discussion from S.L.K., R.M., S.L.Z., and S.X.L. All authors reviewed, edited, and approved the final draft of the manuscript. All authors are accountable for the accuracy and integrity of the work.

\section{COMPETING INTERESTS}

The authors declare no competing interests.

\section{ADDITIONAL INFORMATION}

Supplementary information The online version contains supplementary material available at https://doi.org/10.1038/s41541-021-00412-6. 
Correspondence and requests for materials should be addressed to Sabra L. Klein or Sean X. Leng.

Reprints and permission information is available at http://www.nature.com/ reprints

Publisher's note Springer Nature remains neutral with regard to jurisdictional claims in published maps and institutional affiliations.

Open Access This article is licensed under a Creative Commons Attribution 4.0 International License, which permits use, sharing, adaptation, distribution and reproduction in any medium or format, as long as you give appropriate credit to the original author(s) and the source, provide a link to the Creative Commons license, and indicate if changes were made. The images or other third party material in this article are included in the article's Creative Commons license, unless indicated otherwise in a credit line to the material. If material is not included in the article's Creative Commons license and your intended use is not permitted by statutory regulation or exceeds the permitted use, you will need to obtain permission directly from the copyright holder. To view a copy of this license, visit http://creativecommons. org/licenses/by/4.0/.

(c) The Author(s) 2021 